\title{
A Mean Value Theorem for Linear Functionals
}

\author{
By D. Meek
}

\begin{abstract}
When working out the errors in discretization formulas, one usually hopes to obtain a mean value type of error. This occurs if the associated Peano kernel is a function which does not change sign. In this paper an expansion is developed which will express any error in mean value form, even when the associated Peano kernel is a function which changes sign.
\end{abstract}

1. Introduction. Let the degree of a linear functional $L$ be defined as the nonnegative integer $n$ such that $L\left(x^{i}\right)=0$ for $i=0,1, \ldots, n$, and $L\left(x^{n+1}\right) \neq 0$. Let $\left\{x_{i}\right\}$ be a set of $l$ distinct points $x_{1}<x_{2}<\ldots<x_{l}$ and $a_{i j}$ be a set of $l(q+1)$ coefficients, $i=1,2, \ldots, l$, and $j=0,1, \ldots, q$, then the linear functionals to be considered are of the form

$$
L(y)=\sum_{(i, j) \in E} a_{i j} y^{(j)}\left(x_{i}\right),
$$

where $q \leqslant n$, the degree of $L$, and $E$ is the nonempty set of pairs $(i, j)$ such that $a_{i j} \neq 0$. Assuming that $y(x)$ is infinitely differentiable, $y(x)$ and its derivatives can be expanded about one of the $x_{i}$ points, $e$, and $L(y)$ can be expressed

$$
L(y)=b_{n+1} y^{(n+1)}(e)+b_{n+2} y^{(n+2)}(e)+\ldots, \quad b_{n+1} \neq 0 .
$$

The main result of this paper is the theorem that if the series (1.2) is infinite, then it can always be written as a finite series ending with a derivative evaluated at an unknown point $\xi$ in $\left(x_{1}, x_{l}\right)$.

For example, the linear functional of degree 2

$$
M(y)=4 y(-1)-4 y(1)+y^{\prime}(-1)+6 y^{\prime}(0)+y^{\prime}(1)
$$

has $x_{1}=-1, x_{2}=0, x_{3}=1, q=1$ and

$$
a_{i j}=\left(\begin{array}{rr}
4 & 1 \\
0 & 6 \\
-4 & 1
\end{array}\right)
$$

If $y(x)$ is infinitely differentiable, then $M(y)$ can be written

$$
M(y)=b_{3} y^{(3)}(0)+b_{4} y^{(4)}(0)+\ldots,
$$

where $b_{3}=-1 / 3, b_{4}=0, b_{5}=1 / 60, b_{6}=0, b_{7}=1 / 840, \ldots$ It is easy to show that

$$
M(y)=-\frac{1}{3} y^{(3)}(0)+\frac{1}{60} y^{(5)}\left(\xi_{1}\right), \quad-1<\xi_{1}<1,
$$

Received May 29, 1979.

1980 Mathematics Subject Classification. Primary 41 A58. 
and also

$$
M(y)=-\frac{1}{3} y^{(3)}(0)+\frac{1}{60} y^{(5)}(0)+\frac{1}{840} y^{(7)}\left(\xi_{2}\right), \quad-1<\xi_{2}<1 .
$$

Expansions of this type have been considered ([1] and [3]) for functionals like (1.1) where the values of the nonzero coefficients $a_{i j}$ are chosen to give a functional of maximum degree. The results of this paper apply to all functionals like (1.1).

2. Mean Value Thoerem. The infinite series (1.2) will be developed by integration by parts and it will be shown that this series can always be written as a finite series ending with a derivative evaluated at an intermediate point $\xi$ in $\left(x_{1}, x_{l}\right)$.

According to Peano's theorem ([2] and [6]), the $n$th degree functional (1.1) can be written

$$
L(y)=\int_{x_{1}}^{x_{l}} y^{(n+1)}(t) K(t) d t,
$$

where the kernel $K(t)$ is defined

$$
K(t)=\sum_{(i, j) \in E} \frac{a_{i j}}{(n-j) !}\left(x_{i}-t\right)_{+}^{n-j},
$$

with

$$
\left(x_{i}-t\right)_{+}^{n}= \begin{cases}0 & \text { if } t \geqslant x_{i}, \\ \left(x_{i}-t\right)^{n} & \text { if } t<x_{i},\end{cases}
$$

for $n=0,1,2, \ldots$. In this paper the statement that a function has one sign will mean that the function is nonnegative and is strictly positive over some interval or the function is nonpositive and strictly negative over some interval. If $K(t)$ is a function of one sign for $t \in\left[x_{1}, x_{l}\right]$, then (2.1) can be written

$$
L(y)=\frac{1}{(n+1) !} L\left(x^{n+1}\right) y^{(n+1)}(\xi), \quad x_{1}<\xi<x_{l} .
$$

Using $K(t)$ from (2.2), let

$$
K_{0}(t)=K(t)
$$

and, for $m=1,2,3, \ldots$, let

$$
K_{m}(t)= \begin{cases}-\int_{x_{1}}^{t} K_{m-1}(u) d u, & x_{1} \leqslant t<e, \\ -\int_{x_{l}}^{t} K_{m-1}(u) d u, & e \leqslant t<x_{l}, \\ 0, & \text { other values of } t .\end{cases}
$$

The series (1.2) will now be developed using a variant of Darboux's expansion [4, p. 440]. The linear functional $L(y)$ expressed as in (2.1) can be written

$$
L(y)=-\int_{x_{1}}^{e} y^{(n+1)}(t) d\left\{K_{1}(t)\right\}-\int_{e}^{x_{l}} y^{(n+1)}(t) d\left\{K_{1}(t)\right\},
$$


and integration by parts then gives

$$
L(y)=y^{(n+1)}(e) \int_{x_{1}}^{e} K_{0}(t) d t-y^{(n+1)}(e) \int_{x_{l}}^{e} K_{0}(t) d t+\int_{x_{1}}^{x_{l}} y^{(n+2)}(t) K_{1}(t) d t
$$

or

$$
L(y)=y^{(n+1)}(e) \int_{x_{1}}^{x_{l}} K_{0}(t) d t+\int_{x_{1}}^{x_{l}} y^{(n+2)}(t) K_{1}(t) d t
$$

The expansion can be continued, showing that

$$
b_{n+m}=\int_{x_{1}}^{x_{l}} K_{m-1}(t) d t, \quad m=1,2, \ldots
$$

To see that the series (1.2) can be written as a finite number of terms, it is sufficient to show that there is an $m$ for which $K_{m}(t)$ is a function of one sign on $\left[x_{1}, x_{l}\right]$. This result will be proven in Theorem 2.2.

If the expansion point $e$ is not equal to $x_{1}$ or $x_{l}$, then $K_{m}(t)$ can be separated into two parts by $e$. Define

$$
R_{m}(t)= \begin{cases}K_{m}\left(x_{l}-t\right), & 0<t<x_{l}-e, \\ 0, & \text { otherwise }\end{cases}
$$

and

$$
S_{m}(t)= \begin{cases}K_{m}\left(x_{1}+t\right), & 0<t<e-x_{1} \\ 0, & \text { otherwise }\end{cases}
$$

If the expansion point $e$ is $x_{1}$, then only $R_{m}(t)$ need be defined and if the expansion point $e$ is $x_{l}$, then only $S_{m}(t)$ need be defined. Definitions (2.4) and (2.7) yield

$$
R_{m}(t)=\int_{Q}^{t} R_{m-1}(u) d u, \quad m=1,2,3, \ldots,
$$

and definitions (2.4) and (2.8) yield

$$
S_{m}(t)=-\int_{0}^{t} S_{m-1}(u) d u, \quad m=1,2,3, \ldots
$$

The relations (2.9) and (2.10) are useful in proving certain properties of $R_{m}(t)$ and $S_{m}(t)$.

THEOREM 2.1. For sufficiently large $m, R_{m}(t)$ is a function of one sign with the same sign as $a_{r s}$ in (1.1), where $r$ is the largest first component of elements in $E$, and $s$ is the largest second component of elements in $E$ which have $r$ as a first component.

Proof. According to (2.7), (2.4) and (2.2),

$$
R_{0}(t)=\sum_{(i, j) \in E} \frac{a_{i j}}{(n-j) !}\left(x_{i}-x_{l}+t\right)_{+}^{n-j}, \quad 0<t<x_{l}-e
$$


and using (2.9) $m$ times,

$$
R_{m}(t)=\sum_{(i, j) \in E} \frac{a_{i j}}{(m+n-j) !}\left(x_{i}-x_{l}+t\right)_{+}^{m+n-j}, \quad 0<t<x_{l}-e .
$$

Letting $F$ be the set of numbers which are first components of the members of $E$ and letting $G_{i}$ be the set of numbers which are second components of pairs in $E$ that have $i$ as first component, $R_{m}(t)$ can be rewritten

$$
\sum_{i \in F} \frac{\left(x_{i}-x_{l}+t\right)_{+}^{m+n-J_{i}}}{\left(m+n-J_{i}\right) !}\left\{\sum_{j \in G_{i}} a_{i j} \frac{\left(m+n-J_{i}\right) !}{(m+n-j) !}\left(x_{i}-x_{l}+t\right)_{+}^{J_{i}-j}\right\}
$$

where $J_{i}$ is the largest element of $G_{i}$. If $m$ is sufficiently large, the inner sum of the above expression is as close as desired to $a_{i J_{i}}$ for $0<t<x_{l}-e$. Thus, for $m$ sufficiently large, $R_{m}(t)$ has the same sign as

$$
\sum_{i \in F} a_{i J_{i}} \frac{\left(x_{i}-x_{l}+t\right)_{+}^{m+n-J_{i}}}{\left(m+n-J_{i}\right) !}, \quad 0<t<x_{l}-e .
$$

This expression can be written

$$
\sum_{i \in F} \frac{\left(x_{r}-x_{l}+t\right)_{+}^{m+n-s}}{(m+n-s) !}\left\{a_{i J_{i}} \frac{(m+n-s) !}{\left(m+n-J_{i}\right) !} \frac{\left(x_{i}-x_{l}+t\right)_{+}^{m+n-J_{i}}}{\left(x_{r}-x_{l}+t\right)_{+}^{m+n-s}}\right\}
$$

where $r$ is the largest member of $F$ and $s$ is $J_{r}$ or the largest member of $G_{r}$. If $m$ is sufficiently large, the above sum is as close as desired to

$$
\frac{a_{r s}}{(m+n-s) !}\left(x_{r}-x_{l}+t\right)_{+}^{m+n-s,} \quad 0<t<x_{l}-e,
$$

since, for all $e<x_{i}<x_{r}$,

$$
\lim _{m \rightarrow \infty} \frac{(m+n-s) !}{\left(m+n-J_{i}\right) !} \frac{\left(x_{i}-x_{l}+t\right)_{+}^{m+n-J_{i}}}{\left(x_{r}-x_{l}+t\right)_{+}^{m+n-s}}=0 .
$$

Thus, $R_{m}(t)$ takes the sign of

$$
\frac{a_{r s}}{(m+n-s) !}\left(x_{r}-x_{l}+t\right)_{+}^{m+n-s}
$$

or $a_{r s}$ for $m$ sufficiently large.

THEOREM 2.2. There are infinitely many values of $m$ for which $K_{m}(t)$ is a function of one sign.

Proof. It is clear from Theorem 2.1 that for all $m$ above some value $R_{m}(t)$ is either nonpositive or nonnegative. A similar analysis on $S_{m}(t)$ shows that for all $m$ above some value $S_{m}(t)$ is a function of one sign and that that sign changes each time $m$ increases by one. Thus, there must be an infinite number of values of $m$ for which $K_{m}(t)$ is a function of one sign. The argument also applies, if the expansion point $e$ is $x_{1}$ or $x_{l}$ and only one of $R_{m}(t)$ or $S_{m}(t)$ is defined. 
THEOREM 2.3. There are infinitely many values of $m$ for which the series (1.2) can be written

$$
\begin{aligned}
& L(y)=b_{n+1} y^{(n+1)}(e)+\ldots+b_{n+m} y^{(n+m)}(e)+b_{n+m+1} y^{(n+m+1)}(\xi), \\
& x_{1}<\xi<x_{l} .
\end{aligned}
$$

Proof. Choose any $m$ for which $K_{m}(t)$ is a function of one sign on $\left[x_{1}, x_{l}\right]$. By (2.5) the functional $L$ can be written

$$
L(y)=b_{n+1} y^{(n+1)}(e)+\ldots+b_{n+m} y^{(n+m)}(e)+\int_{x_{1}}^{x_{l}} y^{(n+m+1)}(t) K_{m}(t) d t .
$$

The mean value theorem applied to the integral gives the required result.

3. Example. The operator

$$
M(y)=4 y(-1)-4 y(1)+y^{\prime}(-1)+6 y^{\prime}(0)+y^{\prime}(1)
$$

might arise as a quadrature formula

$$
4 \int_{-1}^{1} u(x) d x=u(-1)+6 u(0)+u(1)+E .
$$

The quantity $E$ can be expressed in several ways, one way being as an infinite series like (1.2). The operator $M(y)$ is of degree 2 and its Peano kernel, $K(t)$, is a function which changes sign. Following a standard procedure $([2],[5],[6],[7])$,

$$
|E| \leqslant \max _{-1 \leqslant x \leqslant 1}\left|u^{(2)}(x)\right| \int_{-1}^{1}|K(t)| d t=\frac{1}{2} \max _{-1<x \leqslant 1}\left|u^{(2)}(x)\right| .
$$

If the method in this paper is followed, then $E$ could be expressed

$$
E=-\frac{1}{3} u^{(2)}(0)+\frac{1}{60} u^{(4)}(\xi), \quad-1<\xi<1,
$$

and

$$
|E| \leqslant \max _{-1<x<1}\left|-\frac{1}{3} u^{(2)}(0)+\frac{1}{60} u^{(4)}(x)\right|
$$

When $u(x)=x^{4}$ then (3.1) gives $|E| \leqslant 6$, while (3.2) gives $|E| \leqslant 2 / 5$ and the value for $E$ is $-2 / 5$. It should be noted that for a function such as $u(x)=e^{20 x}$, formula (3.1) gives a smaller bound on $|E|$ than formula (3.2).

Acknowledgement. The author would like to thank Dr. Peter Taylor, who inspired this work.

Department of Computer Science

The University of Manitoba

Winnipeg, Manitoba, Canada R3T 2N2

1. G. D. BIRKHOFF, "General mean value and remainder theorems with applications to mechanical differentiation and integration," Trans. Amer. Math. Soc., v. 7, 1906, pp. 107-136.

2. P. J. DAVIS \& P. RABINOWITZ, Methods of Numerical Integration, Academic Press, New York, 1975. 
3. D. FERGUSON, "Sufficient conditions for Peano's kernel to be of one sign," SIAM J. Numer. Anal., v. 10, 1973, pp. $1047-1054$.

4. C. A. STEWART, Advanced Calculus, Methuen, London, 1940.

5. A. H. STROUD, "Error estimates for Romberg quadrature," SIAM J. Numer. Anal., v. 2, 1965 , pp. $480-488$.

6. A. H. STROUD, Numerical Quadrature and Solution of Ordinary Differential Equations, Appl. Math. Series, Vol. 10, Springer-Verlag, New York, 1974.

7. R. A. USMANI, "On improving error bounds in the integration of a boundary value problem," Bull. Calcutta Math. Soc., v. 70, 1978, pp. 33-43. 